\title{
TRATAMIENTO DE LA PROLIFERACIÓN ANGIOMATOSA RETINIANA (RAP). ESTUDIO RETROSPECTIVO
}

\author{
TREATMENT OF RETINAL ANGIOMATOUS PROLIFERATION \\ (RAP). A RETROSPECTIVE STUDY
}

\author{
OLEA JL ${ }^{1}$, SASTRE $\mathrm{M}^{2}$, ARAGÓN JA ${ }^{2}$, CARDONA A ${ }^{2}$, MATEOS JM ${ }^{2}$
}

\begin{abstract}
RESUMEN
Objetivo: Evaluar la evolución y respuesta terapéutica de los pacientes con RAP.

Métodos: Estudio retrospectivo de los pacientes con diagnóstico de RAP, atendidos en nuestro servicio entre Marzo/2002 y Agosto/2005. Se analizaron los angiogramas y OCT basales y durante el seguimiento para el diagnóstico y estadiaje del RAP (clasificación de Yannuzzi). Se analizaron 4 terapéuticas diferentes: Termoterapia transpupilar (TTT), Terapia fotodinámica (TFD), tratamiento combinado de TFD con triamcinolona intravítrea (TIV) y, por ultimo, tratamiento combinado de Fotocoagulación directa con láser de argon de la lesión angiomatosa retiniana (FD) más TFD y TIV. Resultados: Se evalúan 15 ojos de 14 pacientes con el diagnóstico de RAP. La edad media fue de 77,5 años. El tiempo medio de seguimiento fue de 15,9 meses, con una media de 2,5 tratamientos por ojo. La agudeza visual final fue peor en $7(46,7 \%)$ pacientes, igual en otros $7(46,7 \%)$ y mejor en 1 $(6,6 \%)$. La mejoría de la agudeza visual, en los diferentes tratamientos evaluados, fue: 2 de 14 (14,2\%) en el grupo de las TTT; 1 de $5(20 \%)$ con la TFD; 2 de $5(40 \%)$ con la combinación de TFD y TIV, y por último, 3 de $5(60 \%)$ con la triple asociación
\end{abstract}

\begin{abstract}
Purpose: To assess the follow-up and response to treatment in patients with RAP.

Methods: A retrospective follow-up study of patients with RAP diagnosed between March 2002 and August 2005. Baseline and subsequent angiograms and optical coherence tomography results were reviewed and RAP classified according to the 3 stages described by Yannuzzi. The changes observed and the best visual acuity were assessed separately for each of the 4 different treatments used: Transpupillary thermotherapy (TTT), Photodynamic therapy (PDT), combined treatment with PDT and Intravitreal triamcinolone (IVT), and combined treatment with PDT, IVT and direct laser photocoagulation of the vascular intraretinal lesion (DLPh.). Results: Fifteen eyes of 14 patients with RAP were studied (mean age, 77.5 years). The mean follow-up was 15.9 months and the mean number of treatments was 2.5 . The final visual acuity was worse in $7(46.7 \%)$, stable in $7(46.7 \%)$ and better in 1 $(6.6 \%)$. Visual acuity improvement, in regard to the treatment used, was: TTT group: 2 out of 14 (14.2\%): PDT group: 1 out of 5 (20\%); PDT + IVT group: 2 out of $5(40 \%)$ and DLPh. + PDT + IVT group: 3 out of $5(60 \%)$.
\end{abstract}

Recibido: 1/2/06. Aceptado: 17/11/06.

Hospital Son Dureta. Islas Baleares. España.

1 Doctor en Medicina.

2 Licenciado en Medicina.

Comunicación presentada en el X Congreso de la Sociedad Española de Retina-Vítreo (Barcelona).

Correspondencia:

José Luis Olea Vallejo

Servicio de Oftalmología. Hospital Son Dureta

C/. Andrea Doria, 55

07014 Baleares

España

E-mail: insaoft@hsd.es 
(TFD+FD+TIV).

Conclusiones: A pesar del mal pronostico final de la RAP, el tratamiento más eficaz en cada brote fue la asociación de terapia fotodinámica, fotocoagulación focal y triamcinolona intravítrea. Mientras que el peor fue la TTT.

Palabras clave: Proliferación angiomatosa retiniana, terapia fotodinámica, degeneración macular asociada a la edad, triamcinolona intravítrea, termoterapia transpupilar.
Conclusions: The final prognosis for RAP, in terms of visual acuity, was generally poor. However the best treatment was the combined treatment with $\mathrm{DLPh}+\mathrm{PDT}+\mathrm{IVT}$, while the worst was TTT (Arch Soc Esp Oftalmol 2007; 82: 27-36).

Key words: Retinal angiomatous proliferation, Intravitreal triamcinolone acetonide, Aging Macular Degeneration, Photodynamic therapy, Transpupillary thermotherapy.

\section{INTRODUCCIÓN}

La proliferación angiomatosa retiniana (RAP) ha sido descrita y sistematizada (1), recientemente, como una variante clínica de la Degeneración macular asociada a la edad (DMAE).

Las mejoras técnicas como la ICG dinámica o la OCT (2) han permitido conocer con más precisión los detalles anatómicos y funcionales de esta patología, caracterizada por la aparición de una lesión angiomatosa retiniana o neovascularización intraretinal que progresa rápidamente, al espacio subretiniano, se suele asociar a un desprendimiento seroso del epitelio pigmentario y concluye con la aparición de una neovascularización coroidea con la que se fusiona a través de gruesas comunicaciones retino-coroideas (3).

En general tiene mal pronóstico y se bilateraliza con mucha frecuencia, (algunas series incluso llegan al $100 \%$ a los 3 años) (4). Se han empleado diferentes tratamientos, solos o asociados, en el manejo de esta enfermedad: terapia fotodinámica (TFD), termoterapia transpupilar (TTT), fotocoagulación directa con láser de argón, fármacos antiangiogénicos y corticoides intravítreos, e incluso cirugía (5-7). Sin embargo, son series pequeñas y todavía no esta sistematizado su manejo.

En nuestro centro se han empleado diferentes tratamientos en el manejo de los brotes o recidivas de los pacientes con RAP. El presente trabajo tiene como finalidad la revisión de estos tratamientos en cuanto a efectividad y complicaciones, con todas las limitaciones que tiene un estudio retrospectivo en una serie pequeña.

\section{MATERIAL Y MÉTODOS}

Estudio retrospectivo de los pacientes con el diagnóstico de RAP que fueron atendidos en nuestro servicio entre marzo/2002 y agosto/2005. La naturaleza retrospectiva del estudio hizo que algunos pacientes fueran descartados por falta de seguimiento o datos incompletos, limitándose el análisis sólo a aquellos con seguimiento adecuado y que acudieron de forma regular a las revisiones.

Los criterios de inclusión, para el diagnóstico de RAP, fueron la asociación de lesión angiomatosa intraretinal en:

- Edad igual o superior a 55 años.

- Signos de DMAE, incluyendo drusas blandas o duras, alteraciones pigmentarias o desprendimiento seroso de epitelio pigmentario, y signos de atrofia de epitelio pigmentario y/o coriocapilar.

- Se excluyeron aquellos pacientes con otras causas, posibles de neovascularización coroidea o retiniana, como miopía degenerativa, estrías angioides, enfermedades infecciosas o inflamatorias coriorretinianas, tumores, enfermedades heredodegenerativas o traumatismos.

- ICG dinámica. Zona hiperfluorescente que se rellena desde vaso retiniano, con alteración de epitelio pigmentario-coriocapilar subyacente. Pudiendo coexistir otras lesiones retinocoroideas.

- OCT. Zona hiperreflectante en capa neurorretinal, asociado o no a desprendimiento de epitelio pigmentario subyacente. Pudiendo coexistir otras lesiones retinocoroideas.

Tras la recogida de la presentación de los síntomas y datos epidemiológicos se efectuó un estudio 
oftalmológico completo: agudeza visual con optotipos estándar de Snellen, examen de polo anterior con lámpara de hendidura, presión intraocular, y exploración de fondo de ojo con cristal de Goldmann o lente Volk Superfield NC. Tras la sospecha, y en un tiempo inferior a 15 días desde la primera exploración, se efectuaron: angiografía dinámica retiniana con fluoresceína y verdeindocianina (HRA Engineering, Heidelberg) y OCT (OCT 3, Zeiss). Se clasificaban en los 3 estadios de la clasificación de Yannuzzi.

En el mismo día se efectuaba el tratamiento, cuya técnica quedaba a discreción del oftalmólogo en función de los hallazgos clínicos y de la técnica más útil a la vista de los conocimientos del momento.

Se han analizado las siguientes terapéuticas utilizadas, de forma aislada o combinada:

- Terapia fotodinámica con verteporfino, se siguieron las recomendaciones standard, si bien se realizaba a continuación de la exploración, aprovechando la vía venosa periférica utilizada para la administración de los colorantes. Como fuente de láser se utilizó el equipo Activis de Quantel Medical (Quantel Mediacal Inc, Clermont-Ferrand, Francia)- BVI que emite láser rojo de $689 \mathrm{~nm}$. Cuando se combinó con fotocoagulación directa, se realizaba primero la TFD y a continuación se aplicaba el láser argón verde (frecuencia $532 \mathrm{~nm}$, desdoblada láser Nd:YAG). Cuando se combinó con Triamcinolona intravítrea, esta se administraba entre 3 y 5 días después de la TFD.

- Termoterapia transpupilar, se administraba con el equipo de Iris Medical (Iris Medical Instruments, Mountain View, CA, USA) que utiliza una fuente de diodo $(810 \mathrm{~nm})$, se usa una técnica subumbral, con lente Mainster de Volk de campo amplio $125^{\circ}$, utilizando la tabla recomendada por el fabricante. El tiempo de administración era fijo de 1 minuto, y cuando se utilizó un diámetro de salida de $3 \mathrm{~mm}$ (diámetro real $4,41 \mathrm{~mm}$ ) la potencia fue de $1094 \mathrm{~mW}$. y cuando se utilizó la de $2 \mathrm{~mm}$ (diámetro real 2,94 mm) la potencia fue de $729 \mathrm{~mW}$.

- Triamcinolona intravítrea. Tres días antes y después utilizaban colirio de cloranfenicol cada 8 horas; en una sala específica, fuera del área quirúrgica y tras anestesia tópica y gotas de Betadine ${ }^{\circledR}$ monodosis [povidona iodada al 10\%] diluido al $50 \%$, se ponía blefarostato, se utilizaban guantes estériles y se inyectaban $0,1 \mathrm{ml}$ (4 mgrs) de Triamcinolona (Trigon depot ${ }^{\circledR}$ ), sin manipulación previa. El paciente estaba bajo observación hasta que cedía el dolor (si lo tenía) o recuperaba visión. Se efectuaba control de la presión intraocular y de la existencia de efectos adversos a la semana.

- Fotocoagulación directa, se administraba con un láser de argón verde (frecuencia $532 \mathrm{~nm}$, desdoblada láser Nd:YAG), se comienza con un tamaño de $100 \mu$ y una intensidad de $100 \mathrm{~mW}$ y tiempo de exposición de $100 \mathrm{msg}$, aumentando los parámetros de intensidad y tiempo de exposición hasta lograr el blanqueamiento de la lesión vascular superficial.

En un paciente, se utilizó fotocoagulación directa con láser infrarojo potenciado con verde Indocianina mas Triamcinolona intravítrea, en uno de los brotes.

El seguimiento del paciente se efectuaba a la semana, en los que se inyectaba Triamcinolona, en los restantes al mes, durante los 3 primeros meses y luego cada 3 meses hasta completar el año. Si se producía una recidiva o aparición de nueva lesión se comenzaba el ciclo nuevamente.

Los resultados se han recogido en una tabla Excel del programa informático Office XP (Microsoft Excel, Microsoft Co, USA), realizándose el estudio descriptivo de las variables cualitativas y cuantitativas.

La agudeza visual se ha evaluado en 5 rangos de visión: inferior a 0,05 , entre 0,05 y 0,1 , entre 0,2 y 0,3 , entre 0,4 y 0,5 , e igual o superior a 0,6 . Interpretando mejoría o empeoramiento un salto entre rangos en uno $\mathrm{u}$ otro sentido. La mejor agudeza visual tras el tratamiento era la utilizada para valorar la efectividad de la técnica terapéutica. Los pacientes fueron seguidos hasta que se obtenían lesiones atróficas o secas, o se consideraban agotadas las posibilidades terapéuticas o siguen en observación, el seguimiento mínimo es de 3 meses.

\section{RESULTADOS}

Se recogen los resultados en 15 ojos de 14 pacientes. Los datos epidemiológicos y de la exploración inicial aparecen en la tabla I. La edad media es de 77,5 (DS 6,7) años.

En la tabla II se muestran la agudeza visual de partida, la final tras los tratamientos administrados y la visión y patología del ojo contralateral (adelfo).

Dado que los pacientes fueron sometidos a distintas técnicas terapéuticas en los brotes, éstos se evalúan por separado para valorar la eficacia de la técnica.

El estadio de las lesiones en el primer tratamiento fueron siete en estadio 1 y ocho en estadio 2 , ha 
Tabla I. Datos epidemiológicos de la serie

\begin{tabular}{|c|c|c|c|c|c|c|c|c|}
\hline $\mathrm{N}^{\mathrm{o}}$ & $\mathrm{S}$ & Edad & Enf. General & $\mathrm{Oj}$ & Cristalino & Pio & TEP. (m) & AVI \\
\hline 1 & $\mathrm{H}$ & 87 & & I & Catarata ++ & 15 & 1 & 0,1 \\
\hline 2 & V & 80 & HTA, Linfoma & I & Pseudo. & $14 *$ & 1,5 & CD $1 \mathrm{~m}$ \\
\hline 3 & $\mathrm{H}$ & 81 & HCL, E. Caro. & I & Catarata + & 15 & 8 & $\mathrm{CD} 1 / 2 \mathrm{~m}$ \\
\hline 4 & $\mathrm{H}$ & 79 & C. Isqué. & D & Pseudo. & 16 & 4 & 0,05 \\
\hline 5 & $\mathrm{H}$ & 76 & & D & Pseudo. & 14 & 1 & 0,7 \\
\hline 6 & $\mathrm{H}$ & 67 & & D & & 23 & 1 & 0,8 \\
\hline 7 & $\mathrm{H}$ & 87 & HTA & D & Pseudo. & 17 & DESC & CD $2 \mathrm{~m}$ \\
\hline 8 & $\mathrm{H}$ & 70 & HTA & D & & 16 & 0,25 & 0,1 \\
\hline 9 & $\mathrm{H}$ & 81 & DM, HTA & I & Pseudo. & 20 & 4 & 0,1 \\
\hline \multirow[t]{2}{*}{10} & $\mathrm{H}$ & 69 & HTA & I & & 17 & 0,25 & 0,4 \\
\hline & & & & D & & 15 & 0,5 & 0,6 \\
\hline 11 & V & 76 & & I & Catarata + & 18 & 1 & 0,1 \\
\hline 12 & $\mathrm{H}$ & 85 & EPOC & I & Catarata + & 16 & 4 & 0,2 \\
\hline 13 & $\mathrm{H}$ & 69 & HTA & I & & 18 & 2 & 0,2 \\
\hline 14 & $\mathrm{H}$ & 79 & HTA, Valvulop. & D & & 23 & 7 & 0,3 \\
\hline
\end{tabular}

N. ${ }^{\text {: }}$ N. Paciente; S: Sexo; H: Hembra; V: Varón; Oj.: Ojo; Enf. General: Enfermedad general asociada relevante; HTA: Hipertensión arterial; E. Caro.: Estenosis carotidea; HCL: Hipercolesterolemia; C. Isqué.: Cardiopatía Isquémica; DM: Diabetes Mellitas; EPOC: Broncopatía Obstructiva crónica; Valvulop.: Valvulopatía cardíaca; Pseudo.: Pseudofaquia; Pio: Presión intraocular en milímetros de mercurio; TEP.: Tiempo de sintomatología previa en meses; AVI: Agudeza visual inicial; CD: Cuenta dedos en metros; * El paciente n. ${ }^{\circ} 2$ tenía un glaucoma crónico simple controlado con tratamiento médico.

medida que avanzaba en número de brotes aumentaban los estadios más avanzados. En total y sumando todos los brotes tratados hubo: nueve estadios 1, diecisiete estadios 2 y doce estadios 3 .

La terapia fotodinámica se utilizó en 18 brotes: cinco de forma aislada, tres asociado a fotocoagulación directa, cinco asociado a triamcinolona intravítrea y cinco con triamcinolona intravítrea y fotocoagulación directa. Los resultados aparecen en la tabla III.
La termoterapia transpupilar se efectuó en 14 brotes de forma aislada y en dos asociada a triamcinolona intravítrea. Los resultados aparecen en la tabla IV.

En una ocasión se han utilizado como único tratamiento en ese brote: triamcinolona intravítrea, fotocoagulación directa más triamcinolona intravítrea, fotocoagulación directa en vaso aferente con diodo potenciado con verde indocianina más triamcinolona intravítrea, y sólo fotocoagulación directa.

Tabla II. Tratamientos y resultados

\begin{tabular}{|c|c|c|c|c|c|c|}
\hline N. ${ }^{o}$ & AVI & N. ${ }^{\circ}$ TTOs & Segui. (m.) & AVF & AV Adelfo & Pat. Adel. \\
\hline 1 & 0,1 & 3 & 15 & CD $1 \mathrm{~m}$. & $\mathrm{CD} 1 / 2 \mathrm{~m}$ & DMAE h. \\
\hline 2 & CD $1 \mathrm{~m}$. & 1 & 4 & $\mathrm{CD} 1 / 2 \mathrm{~m}$ & 0,7 & \\
\hline 3 & $\mathrm{CD} 1 / 2 \mathrm{~m}$ & 1 & 3 & $\mathrm{CD} 11 / 2 \mathrm{~m}$ & 0,5 & Catarata \\
\hline 4 & 0,05 & 1 & 19 & 0,05 & 0,05 & DMAE h. \\
\hline 5 & 0,7 & 5 & 25 & 0,1 & 0,05 & DMAE h. \\
\hline 6 & 0,8 & 1 & 11 & 0,7 & 0,5 & DMAE atro \\
\hline 7 & CD $2 \mathrm{~m}$ & 3 & 26 & $\mathrm{CD} 1 / 2 \mathrm{~m}$ & 0,7 & \\
\hline 8 & 0,1 & 1 & 15 & M.M. & 1 & \\
\hline 9 & 0,1 & 2 & 17 & 0,05 & $\mathrm{CD} 1 / 2 \mathrm{~m}$ & D.R. \\
\hline \multirow[t]{2}{*}{10} & 0,4 & 2 & 23 & $\mathrm{CD} 1 / 2 \mathrm{~m}$ & 0,2 & DMAE h. \\
\hline & 0,6 & 5 & 18 & 0,2 & $\mathrm{CD} 1 / 2 \mathrm{~m}$ & DMAE h. \\
\hline 11 & 0,1 & 2 & 5 & 0,1 & M.M. & DMAE h. \\
\hline 12 & 0,2 & 4 & 24 & $\mathrm{CD} 1 / 2 \mathrm{~m}$ & M.M. & DMAE h. \\
\hline 13 & 0,2 & 1 & 3 & 0,6 & 0,1 & DMAE h. \\
\hline 14 & 0,3 & 6 & 33 & 0,05 & M.M. & DMAE h. \\
\hline
\end{tabular}

N. ${ }^{\text {: }}$ N. ${ }^{o}$ Paciente; AVI: Agudeza visual Inicial; N. ${ }^{\circ}$ TTOs: Número de tratamientos; AVF: Agudeza visual Final; AV Adelfo: Agudeza visual ojo adelfo; Pat. Adel.: Patología ojo adelfo; CD: Cuenta dedos (en metros); M.M.: Movimiento de manos; DMAE atro.: Degeneración macular atrófica; DMAE h.: Degeneración macular asociada a la edad húmeda o cicatricial o RAP. 
Tabla III. Tratamientos con terapia fotodinámica, sola 0 combinada

\begin{tabular}{lccc}
\hline Estadio & AVP & Tratamiento & MAV \\
\hline 2 & 0,1 & TFD1 & 0,3 \\
2 & 0,7 & TFD1 & 0,4 \\
2 & 0,1 & TFD1 & CD $1 \mathrm{~m}$ \\
2 & 0,2 & TFD1 & CD 1 m \\
2 & 0,3 & TFD1 & 0,3 \\
2 & CD $1 \mathrm{~m}$. & TFD1 + FD1 & CD 1/2 m \\
2 & CD 1/2 m. & TFD1 + FD1 & CD 1 m \\
2 & 0,05 & TFD1 + FD1 & 0,05 \\
3 & 0,1 & TFD2 + TIV2 & 0,1 \\
2 & 0,1 & TFD3 + TIV3 & 0,4 \\
3 & 0,1 & TFD3 + TIV3 & 0,1 \\
2 & 0,1 & TFD2 + TIV2 & 0,5 \\
3 & 0,05 & TFD3 + TIV2 & CD 1 m \\
2 & 0,05 & TFD2 + TIV2 + FD3 & 0,4 \\
1 & 0,4 & TFD1 + TIV1 + FD2 & 0,5 \\
2 & 0,1 & TFD2 + TIV1 + FD1 & 0,2 \\
1 & 0,8 & TFD1 + TIV1 + FD1 & 0,8 \\
1 & 0,2 & TFD1 + TIV1 + FD1 & 0,6 \\
\hline \hline
\end{tabular}

AVP: Agudeza visual previa; MAV: Mejor agudeza visual postratamiento; TFD: Terapia Fotodinámica; TIV: Triamcinolona intravítrea; FF: Fotocoagulación focal con láser de argón; CD: Cuenta dedos, en metros. El número al lado de la técnica indica el n. ${ }^{\circ}$ acumulado de ese tratamiento.

Sólo se analizarán los resultados de las técnicas utilizadas, al menos en 5 ocasiones.

Evaluación de la agudeza mejor agudeza visual tras el tratamiento:

Tabla IV. Tratamientos con TTT, sola o combinada

\begin{tabular}{lccc}
\hline Estadio & AVP & Tratamiento & MAV \\
\hline Estadio & AVP & TRATAMIENTO & MAV \\
2 & CD 2 m & TTT1 & CD 2 m \\
1 & 0,1 & TTT1 & 0,2 \\
1 & 0,4 & TTT1 & 0,2 \\
2 & 0,2 & TTT1 & 0,2 \\
2 & 0,05 & TTT2 & 0,1 \\
1 & 0,3 & TTT1 & 0,3 \\
2 & 0,1 & TTT1 & 0,1 \\
3 & CD $1 \mathrm{~m}$ & TTT2 & CD $1 \mathrm{~m}$ \\
1 & 0,5 & TTT1 & 0,6 \\
3 & 0,1 & TTT2 & 0,1 \\
2 & 0,2 & TTT2 & 0,2 \\
3 & CD $1 / 2 \mathrm{~m}$ & TTT3 & CD 1/2 m \\
2 & 0,2 & TTT1 & 0,3 \\
2 & 0,3 & TTT2 & 0,2 \\
3 & 0,1 & TTT3 + TIV1 & 0,2 \\
2 & 0,1 & TTT1 + TIV1 & 0,2 \\
\hline \hline
\end{tabular}

AVP: Agudeza visual previa; MAV: Mejor agudeza visual postratamiento; TFD: Terapia Fotodinámica; TIV: Triamcinolona intravítrea; CD: Cuenta dedos, en metros; El número al lado de la técnica indica el n. ${ }^{\circ}$ acumulado de ese tratamiento.
- Terapia fotodinámica: mejora en un caso, estable en un y empeora en tres.

- Terapia fotodinámica y triamcinolona intravítrea: mejora en dos, estable en dos y empeora en uno.

- Terapia fotodinámica con triamcinolona intravítrea y fotocoagulación directa: mejora en tres y estable en dos.

- Termoterapia transpupilar: mejora en dos, estable en once y empeora en un caso.

De los 14 pacientes de la serie, la triamcinolona intravítrea se utilizó en siete pacientes (siete ojos), otro paciente rechazo su uso y por ultimo en otro no se podía usar por padecer glaucoma, se analizan las siguientes complicaciones:

Se pusieron 15 intravítreas en los siete ojos (en dos casos uno, en dos casos dos y en tres casos tres), no produciéndose ninguna endoftalmitis.

En 3 ojos $(42,8 \%)$ se produjo un glaucoma cortisónico controlándose con tratamiento médico.

Por último, dos ojos eran pseudofacos y cinco fáquicos, de estos, al final del seguimiento se indico cirugía de la catarata en dos, que rechazaron ante el mal pronostico visual final y en otro progreso la catarata.

\section{DISCUSIÓN}

La RAP es una variante clínica de la DMAE descrita y sistematizada recientemente (1), cada vez con mayor frecuencia tenemos que enfrentarnos a este problema en la practica clínica diaria, sin embargo existen pocas publicaciones que estandaricen el manejo de esta patología.

Se caracteriza por la aparición de una proliferación vascular intraretinal yuxta o extrafoveal, con un gran componente edematoso-exudativo, y que se acompaña, en el 75-80\% de los casos, de un desprendimiento seroso de epitelio pigmentario (DSEP) subyacente y en un $30 \%$ se acompaña también de un desprendimiento seroso del neuroepitelio (DSNE) adyacente (2,3).

A pesar de su origen yuxta o extrafoveal se caracteriza por su mal pronóstico $(1,3,7)$; en nuestra serie se aprecian como, al final del seguimiento, el $80 \%$ de los ojos queda con una agudeza visual inferior a 0,1 y se produce una pérdida de visión con respecto a la agudeza visual inicial. Teniendo en cuenta que el seguimiento medio ha sido de 15,9 (DS 9,5) meses y la media de tratamientos de 2,5 (DS 1,7), el 
resultado funcional, con respecto los recursos utilizados para su manejo, han sido decepcionantes, el $46,7 \%$ perdió visión, el $46,7 \%$ se estabilizó y solo un $6,6 \%$ mejoró.

La otra característica es su alta frecuencia de bilateralización, frente al $43 \%$ de bilateralización en el conjunto de las DMAE (8), la RAP lo hace en el $40 \%$ al año, el $52 \%$ a los 2 años y el $100 \%$ a los 3 años (3). El 65\% de nuestros pacientes tenían una DMAE húmeda avanzada o RAP en el ojo contralateral, siendo la causa de que la mayoría de los ojos contralaterales tuvieran, también, una visión inferior a 0,1 (fig. 1).

Se han utilizado múltiples tratamientos quirúrgicos y médicos para la DMAE húmeda; en el RAP se ha utilizado, en pacientes aislados, la sección de los vasos aferentes y eferentes de la neovascularización intraretiniana (6); sin embargo, su naturaleza múltiple y recidivante, hace que la cirugía no sea una opción lógica, aunque podría estar indicada en algún caso.

La terapia fotodinámica ha sido utilizada en la RAP, en su serie de trece casos, Boscia (9) obtiene oclusión de la lesión y aplanamiento del DSEP en tres $(23 \%)$ y como complicación tuvo una rotura de epitelio pigmentario en tres casos con grandes DSEP, algo parecido ocurre en la serie de Bottoni (5) donde solo el $11 \%$ (en estadio 2) se inactivan. En nuestra serie (tabla III), se han utilizado TFD en cinco casos, todos en estadio 2 y sólo uno mejora visón y otro se estabiliza. La TFD, de forma aislada, no es un tratamiento muy eficaz en esta patolo-

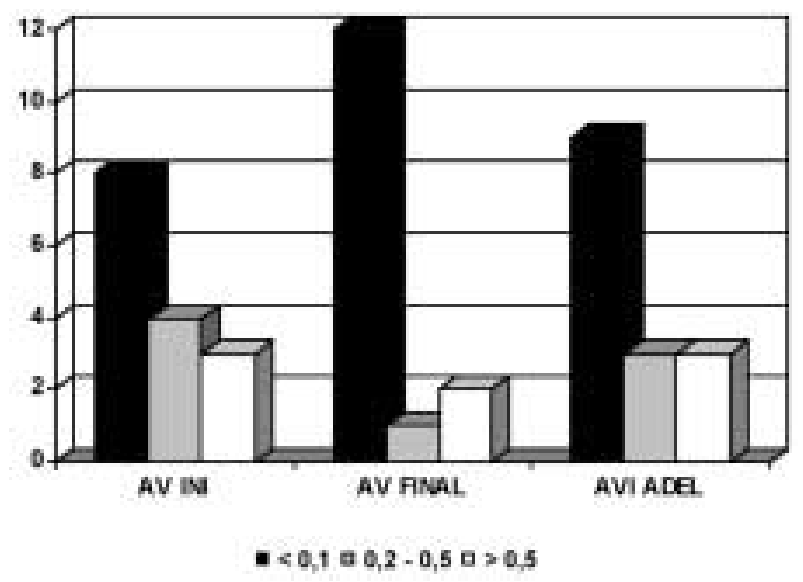

Fig. 1: Agudeza visuales inicial, final y del ojo adelfo. Puede verse que la columna de las visiones más bajas es la más numerosa en los 3. gía, esto podría explicarse porque por su mecanismo de acción, tiene su utilidad en neovasos de origen coroideo, especialmente en la forma clásica, sin embargo en esta patología, en los primeros estadios, la lesión neovascular es puramente intraretiniana.

La termoterapia transpupilar tiene la ventaja de conservar la capa de fibras, y tiene mejor penetración a través de las hemorragias subretinianas (10), teniendo en cuenta que el $94 \%$ de las RAP llevan asociadas hemorragias retinianas (3) y teniendo en cuenta la publicación de Gass en la que aseguraba que en estos casos existía siempre una membrana oculta (11), nosotros aplicamos esta técnica en los primeros casos. Se utilizó en 14 brotes, de forma aislada, y sólo dos casos mejoraron la visión (14\%). Bottoni trata en su serie sólo cinco casos (5) y logra obliteración en dos (en estadio 2), aunque en nuestra serie solo evaluamos agudeza visual, no mejoró ninguno de los siete casos en estadio 2, tratados. En general, la TTT no es una técnica indicada en la RAP, y las ideas y clasificación de Gass no han sido corroboradas.

El láser de argón, se ha demostrado como la técnica más eficaz en el estadio 1, con un $73 \%$ de obliteraciones, sin embargo en estadio 2 la tasa baja al $17 \%$ a pesar de que el tratamiento precoz de la enfermedad (figs. 2-6), mejora los resultados (5); la fotocoagulación directa es imprescindible en el estadio 1. También se ha utilizado el láser como rejilla, o fotocoagulando el vaso aferente de la neovascularización intrarretiniana.

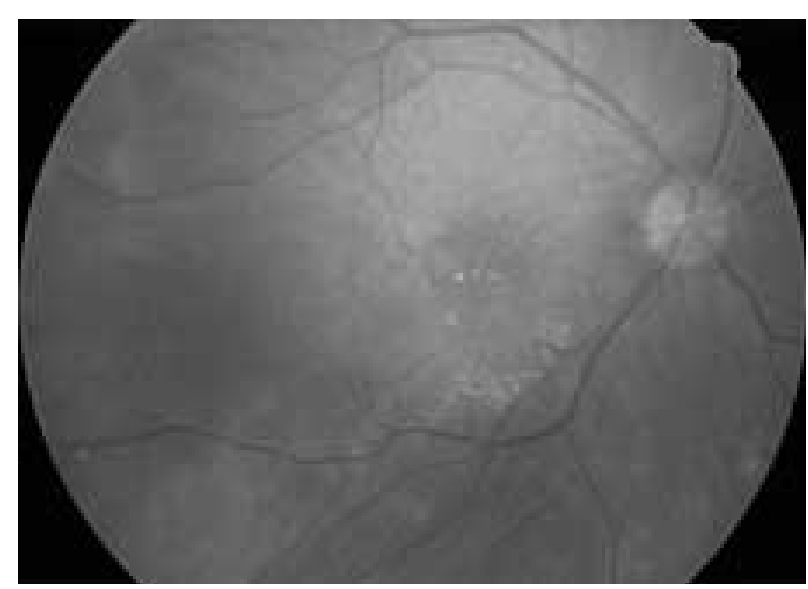

Fig. 2: Caso clínico. Estadio II incipiente. Retinografía OD. Pueden verse exudados en disposición circinada y edema retiniano. 


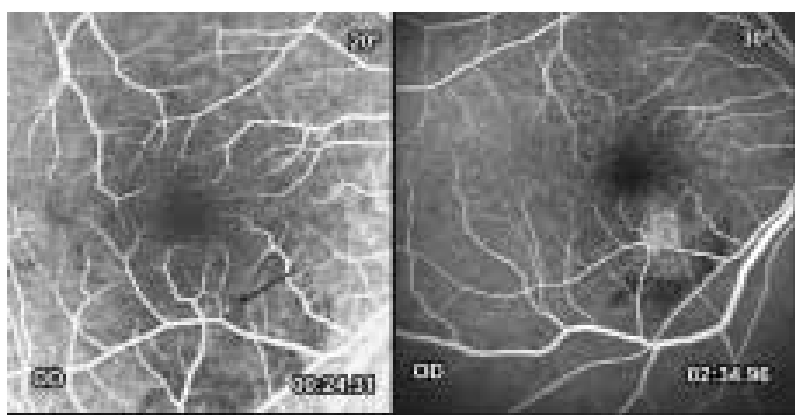

Fig. 3: Angiofluoresceingrafía del caso clínico. Puede verse un pequeño vaso con gran salida de colorante (flecha negra), que aumenta en tiempos tardios.

La triamcinolona intravítrea (TIV) se ha utilizado en el tratamiento de la DMAE por su efecto reparador de la barrera hematorretiniana interna y antiangiogénico $(12,13)$, mejora el edema macular quístico asociado y disminuye el tamaño de la membrana y el número de retratamientos cuando se asocia a TFD. Una simple dosis dura 3-4 meses, por lo que se recomienda su uso combinado (14); en nuestra serie se ha utilizado de forma aislada, y combinado con TTT, TFD y TFD más fotocoagulación directa. El mayor inconveniente reside en las complicaciones: en nuestra serie, no hubo ninguna endoftalmitis en las 15 inyecciones intraoculares, sin embargo de los siete ojos en los que se usaron, tres tuvieron hipertensión ocular controlada con tratamiento medico $(42,8 \%)$, este porcentaje es similar otras series en DMAE como la de Jonas (13) con un 49,3\% (dosis 25 mgrs), y la de Gillies (12) con un $41 \%$ (dosis 4 mgrs). Por último, la catarata, que sí es una complicación importante en otras indicacio-

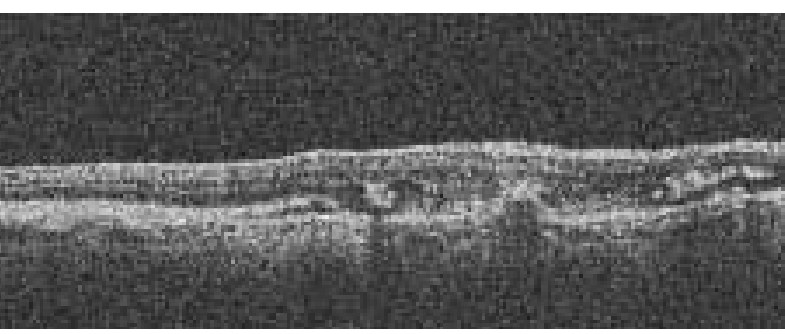

Fig. 5: OCT, corte horizontal de $5 \mathrm{~mm}$. a nivel de la lesión (marcado en la figura 5). Se ve una pequeña zona de desprendimiento de epitelio pigmentario con una zona hiperrefringente adyacente.

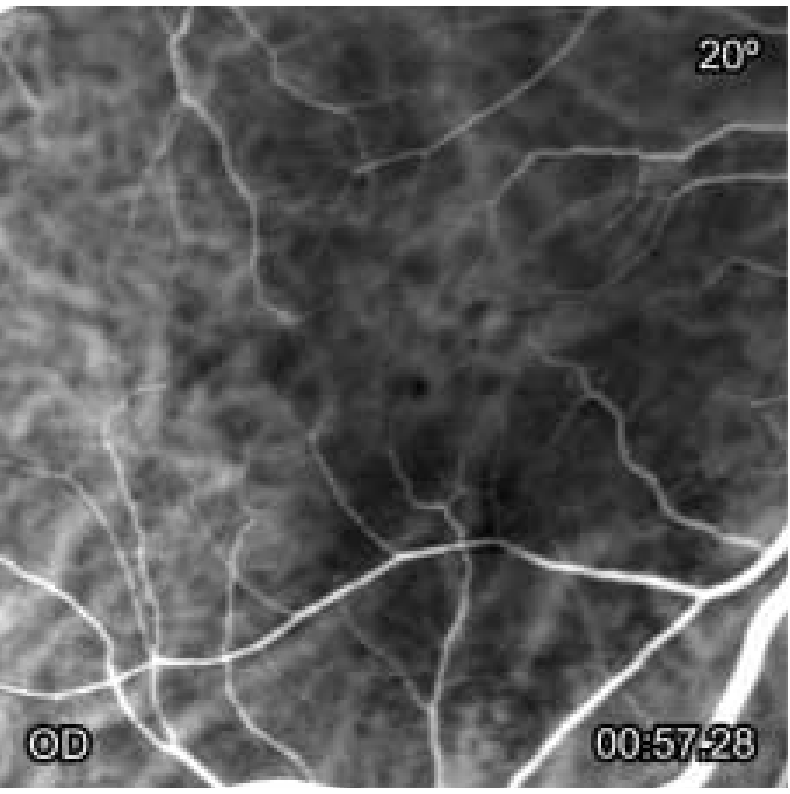

Fig. 6: Verde Indocianina del caso clínico 2 meses después (tiempo precoz). Vaso ocluido tras un tratamiento combinado, que incluyó la fotocoagulación.

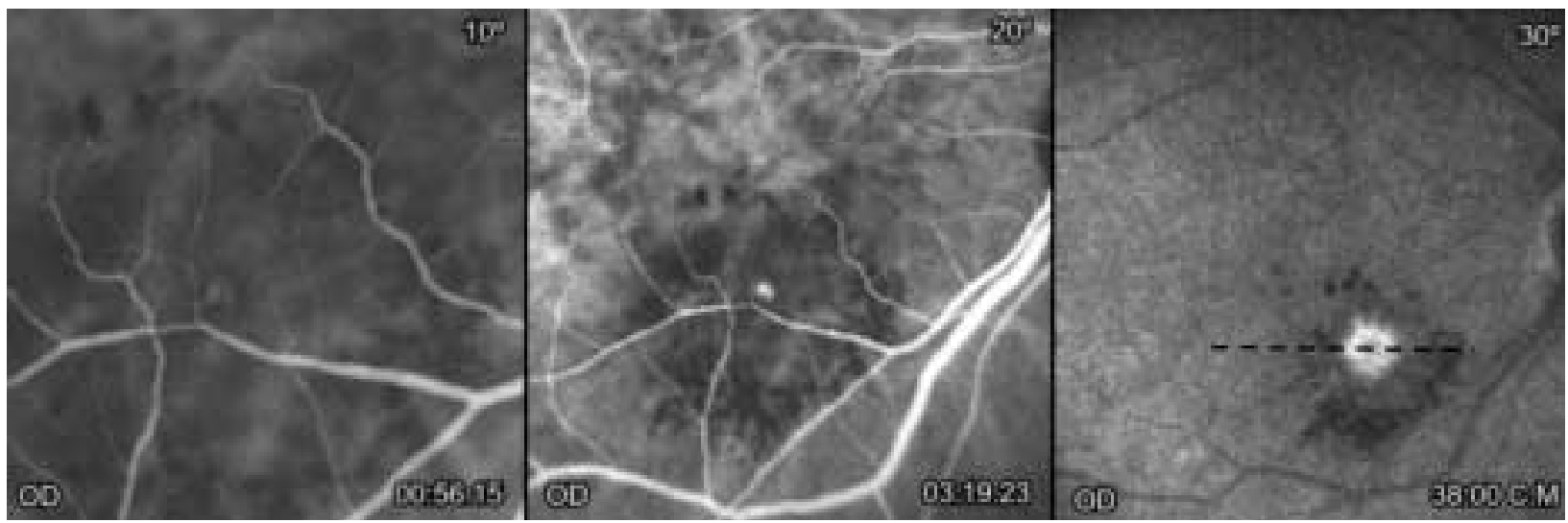

Fig. 4: Verde Indocianina. Puede verse el vaso, en la imagen de $10^{\circ}$. En tiempos tardios es hiperfluorescente por la existencia de fibrina que se tiñe con el VIC. 
nes, en la edad senil, no lo es tanto, porque algunos son ya pseudofacos o la cirugía a esta edad es fácilmente asumida, se indicó cirugía en dos de nuestros cinco pacientes fáquicos y en uno más progresó la catarata preexistente.

Los tratamiento combinados, son los que mejores resultados han ofrecido. Sólo podemos comentar dos asociaciones, porque al menos se efectuaron en 5 ocasiones: la asociación de TFD más TIV se empleo en brotes con estadios 2 y 3, lográndose mejoría de agudeza visual en dos.

Los mejores resultados se obtuvieron con la asociación TFD más TIV más fotocoagulación directa, se empleo en estadios 1 y 2 , y si bien es cierto que los mejores resultados se obtienen en estadios precoces (5), la diferencia es muy grande, en cuatro de los cinco tratamientos la visión mejoró. A pesar de que las agudezas visuales de partida son mejores en este grupo, esto no debe influir y es valida la comparación, ya que en el análisis global de la serie (fig. 7 ), cuanta más agudeza visual más pierden. Tal vez solo la fotocoagulación directa y el Trigon intravítreo fueran suficientes en el estadio 1, nosotros no lo hemos analizado, pero la asociación con terapia fotodinámica ha sido la técnica más eficaz de las empleadas. Probablemente actúen de forma diferente y sinergista, la fotocoagulación directa oblitera, por efecto térmico, la neovascularización intraretinal, la terapia fotodinámica puede actuar sobre el componente intraretinal y/o subretinal si existe, así como la coroides, contribuyendo a resolver el desprendimiento de epitelio pigmentario y por ulti-

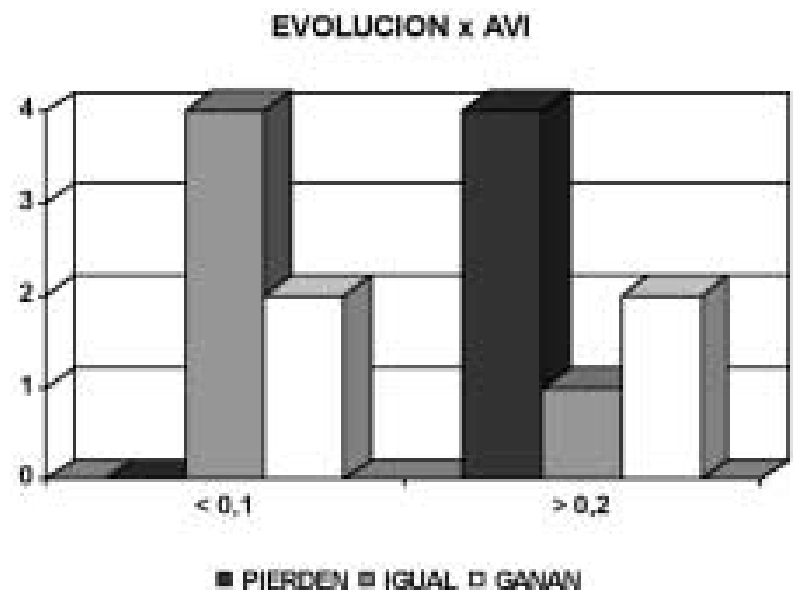

Fig. 7: Resultados globales, según la visión inicial. Los ojos que comienzan con mejor visión son los que más pierden. mo la triamcinolona que actuaría como antiangiogénico y disminuyendo la exudación perilesional tan importante en la RAP.

A falta de la experiencia con los nuevos antiVEGF intravítreos o peribulbares, la asociación TFD más TIV más fotocoagulación directa es la más útil en el manejo de la RAP, en estadio 1 y 2. Por el contrario, la técnica menos eficaz, en cualquiera de los 3 estadios es la TTT.

\section{BIBLIOGRAFÍA}

1. Yannuzzi L, Negrao S, Iida T, Carvalho C, RodriguezColeman H, Slater J, et al. Retinal angiomatous proliferation in age-related macular degeneration. Retina 2001; 21: 416-434.

2. Brancato $R$, Introini $U$, Pierro $L$, Setaccioli $M$, Forti $M$, Bolognesi $G$, et al. Optical coherence tomography (OCT) angiomatous prolifieration (RAP) in retinal. Eur J Ophthalmol 2002; 12: 467-472.

3. Gross NE, Aizman A, Brucker A, Klancnik JM Jr, Yannuzzi LA. Nature and risk of neovascularization in the fellow eye of patients with unilateral retinal angiomatous proliferation. Retina 2005; 25: 713-718.

4. Liu M, Regillo CD. A review of treatments for macular degeneration: a synopsis of currently approved treatments and ongoing clinical trials. Curr Opin Ophthalmol 2004; 15: $221-226$.

5. Bottoni F, Massacesi A, Cigada M, Viola F, Musicco I, Staurenghi $G$. Treatment of retinal angiomatous proliferation in age-related macular degeneration: a series of 104 cases of retinal angiomatous proliferation. Arch Ophthalmol 2005; 123: 1644-1650.

6. Borrillo JL, Sivalingam A, Martidis A, Federman JL. Surgical ablation of retinal angiomatous proliferation. Arch Ophthalmol. 2003; 121: 558-561.

7. Slakter JS, Yannuzzi LA, Schneider U, Sorenson JA, CiardeIla A, Guyer DR, et al. Retinal choroidal anastomoses and occult choroidal neovascularization in age-related macular degeneration. Ophthalmology 2000; 107: 742-753.

8. Bressler NH, Bressler SB, Congdon NG, Ferris FL 3rd, Friedman DS, Klein $R$, et al. Potencial public health impact of Age-Related Eye Disease Study Results. AREDS report no. 11. Arch Ophthalmol 2003; 121: 1621-1624.

9. Boscia F, Furino C, Sborgia L, Reibaldi M, Sborgia C. Photodynamic therapy for retinal angiomatous proliferations and pigment epithelium detachment. Am J Ophthalmol 2004; 138: 1077-1079.

10. Thach AB, Sipperley JO, Dugel PU, Sneed SR, Park DW, Cornelius J. Large-spot size transpupillary thermotherapy for the treatment of occult choroidal neovascularization associated with are-related macular degeneration. Arch Ophthalmol 2003; 121: 817-820.

11. Gass JD, Agarwal A, Lavina AM, Tawansy KA. Focal inner retinal hemorrhages in patients with drusen: an early sign of occult choroidal neovascularization and chorioretinal anastomosis. Retina 2003; 23: 741-751.

12. Gillies MC, Simpson JM, Luo W, Penfold P, Hunyor AB, 
Chua W, et al. A randomized clinical trial of a single dose of intravitreal triamcinolone acetonide for neovascular age-related macular degeneration: one-year results. Arch Ophthalmol 2003; 121: 667-673.

13. Jonas JB, Kreissig I, Hugger P, Sauder G, Panda-Jonas $S$, Degenring R. Intravitreal triamcinolone acetonide for exudative age related macular degeneration. $\mathrm{Br} J$ Ophthalmol 2003; 87:462-468.

14. Spaide RF, Sorenson J, Maranan L. Combined photodynamic therapy with verteporfin and intravitreal triamcinolone acetonide for choroidal neovascularization. Ophthalmology 2003; 110: 1517-1525. 